\title{
Adsorption of noble gas on various adsorbents at cryogenic temperatures
}

\author{
Takashi Shinozaki \\ Kyushu University \\ takashi@nucl.kyushu-u.ac.jp
}

\author{
Kenzo Munakata \\ Kyushu University \\ kenzo@nucl.kyushu-u.ac.jp
}

\section{Hirohumi Okabe \\ Kyushu University}

\section{INTRODUCTUION}

Understanding of adsorption characteristics of $\mathrm{Kr}$ in air on adsorbents used at cryogenic temperature is required to design the monitoring system or recovery system of radioactive noble gases from nuclear off-gases. In this study, the authors conducted a screening test of adsorbents for adsorption of $\mathrm{Kr}$ to search for effective adsorbents. For the adsorbents selected in the screening test, more detailed adsorption characteristics of $\mathrm{Kr}$ were investigated.

\section{EXPERIMENTAL}

All the experiments were conducted by the breakthrough method. In the screening test, a tubular container made of quartz glass, which was charged with adsorbents, was placed in a constant temperature bath filled with liquid argon $(87 \mathrm{~K})$. In the case of more detailed study on adsorption characteristics, a cryostat system was used to control the temperature of adsorbents at various cryogenic temperatures. In this case, adsorbents were charged in a container made of aluminum, which was attached to the cryopanel in the cryostat system. As major component of air is nitrogen, nitrogen containing a certain amount of krypton was introduced to the container of the adsorbents in these experiments. The gas composition of the inlet and outlet streams of the adsorption bed was analyzed with a quadrupole mass spectrometer (Mini-Lab by MKS Inc.). The adsorbents used were preheated under nitrogen atmosphere prior to use.

\section{RESULTS AND DISCUSSION}

Figure 1 shows the amounts of $\mathrm{Kr}$ adsorbed on various adsorbents as the function of partial pressure of Kr. It was found that carbon based adsorbents have better performance for adsorption of $\mathrm{Kr}$ and that the Ambersorb 572 adsorbent has the largest adsorption capacity. It was also found that the adsorption capacity of non-carbon based adsorbents is much lower than that of carbon-based adsorbents.
More detailed adsorption characteristics were investigated for carbon-based adsorbents using the cryostat system. In the temperature range of 90-200 K, the Ambersorb 572 adsorbent has the largest adsorption capacity for the adsorption of $\mathrm{Kr}$ under nitrogen atmosphere. In addition, the effect of temperature change on the adsorption capacity was found to be largest for the Ambersorb 572 adsorbent and smallest for the Kishida-produced activated charcoal adsorbent. The experimental isotherms of carbon-based adsorbents were correlated with the Langmuir or Henry equation, and the adsorption amounts of $\mathrm{Kr}$ at the partial pressure of $0.1 \mathrm{~Pa}$, which corresponds to the atmospheric partial pressure of $\mathrm{Kr}$, and at the temperatures of 80,90 and $100 \mathrm{~K}$ were estimated using the equations.

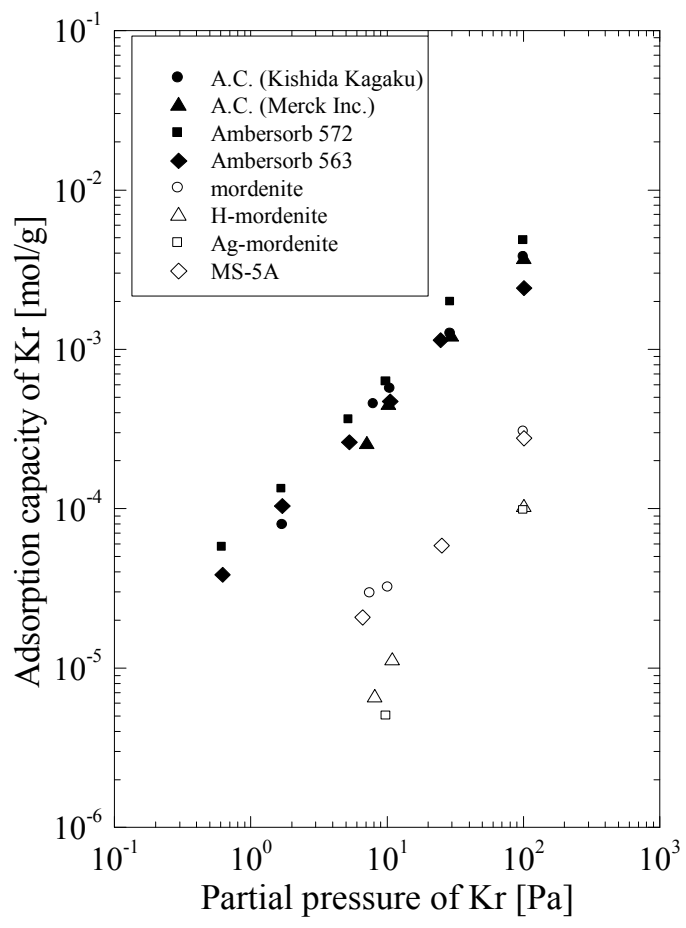

Fig. 1 Adsorption isotherm of $\mathrm{Kr}$ on various adsorbent at liquid argon temperature 


\title{
Adsorption of noble gas on various adsorbents at cryogenic temperatures
}

\section{(DRAFT)}

\author{
Takashi Shinozaki \\ Department of Advanced \\ Energy Engineering Science \\ Interdisciplinary Graduate \\ School of Engineering Science, \\ Kyushu University \\ takashi@nucl.kyushu-u.ac.jp
}

\author{
Kenzo Munakata \\ Department of Advanced \\ Energy Engineering Science \\ Interdisciplinary Graduate \\ School of Engineering Science, \\ Kyushu University \\ kenzo@nucl.kyushu-u.ac.jp
}

\author{
Hirohumi Okabe \\ Department of Advanced \\ Energy Engineering Science \\ Interdisciplinary Graduate \\ School of Engineering Science, \\ Kyushu University
}

Key words: Monitoring, Nuclear reprocessing, Krypton, Adsorption, Adsorbent

\begin{abstract}
Most of ${ }^{85} \mathrm{Kr}$ in atmosphere is released from anthropogenic sources such as tests of nuclear weapons and nuclear reactors. As a chemically inert gas with a long half-life, ${ }^{85} \mathrm{Kr}$ will continue to accumulate in the environment. Thus, it is necessary to monitor the background level of the atmospheric ${ }^{85} \mathrm{Kr}$ concentrations. In this study, the authors conducted a screening test of several adsorbents to search for more suitable one for adsorption of $\mathrm{Kr}$ at under nitrogen atmosphere, which is used for the monitoring system of ${ }^{85} \mathrm{Kr}$. A screening test was carried out by focusing on adsorption characteristics at a cryogenic temperature. With regard to adsorbents selected in the screening test, more detailed adsorption characteristics were examined. As a result, it was found that carbon based adsorbents have better performance for adsorption of $\mathrm{Kr}$ and that the
\end{abstract}

Ambersorb 572 adsorbent has the largest adsorption capacity. It was also found that the adsorption capacity of non-carbon based adsorbents is much lower than that of carbon-based adsorbents.

\section{INTRODUCTUION}

Krypton-85 is a radioactive noble gas that decays via emission of beta $(0.4 \%$ of beta decay emit gamma ray.). At present, the major sources of ${ }^{85} \mathrm{Kr}$ are the nuclear fuel reprocessing plants in Europe (Hirota, M., et al., 2004). As a chemically inert gas with a long half-life (10.7 years), ${ }^{85} \mathrm{Kr}$ will continue to accumulate in the environment. In the monitoring system used at the Meteorological Research Institute in Tsukuba, Japan, atmospheric ${ }^{85} \mathrm{Kr}$ is first collected from air at several sampling stations located in Japan using an adsorption bed immersed in liquid nitrogen, which is followed by chromatographic separation and activity 
counting with a GM tube. There is a problem related to the monitoring system; the adsorption characteristics of $\mathrm{Kr}$ in air on an activated charcoal adsorbent used at cryogenic temperature are still not well understood. In previous works, the authors studied the adsorption of noble gases on several adsorbents (Munakata et al., 1999 and Munakata et al., 2001). A screening test of adsorbents for $\mathrm{Kr}$ was conducted as well (Munakata et al., 2000 and Ianovski et al., 2002). However, in these studies, helium was used as the carrier gas since the major purpose was to obtain basic adsorption characteristics of noble gases on adsorbents. Additionally, the data were taken at relatively higher temperatures because cryogenic operation was out of target. The gas introduced to the adsorption bed used for the monitoring system or recovery of radioactive noble gases from nuclear off-gases is air in the actual process, and thus the effect of coexistent nitrogen on the adsorption of noble gases needs be investigated. Thus, in this study, the authors conducted a screening test of adsorbents for adsorption of $\mathrm{Kr}$ at under nitrogen atmosphere. The screening test was carried out by focusing on adsorption characteristics at liquid argon temperature. Search for more effective adsorbents is important for secure recovery of ${ }^{85} \mathrm{Kr}$ from the atmosphere, which can guarantee the accuracy of the monitoring. Then,

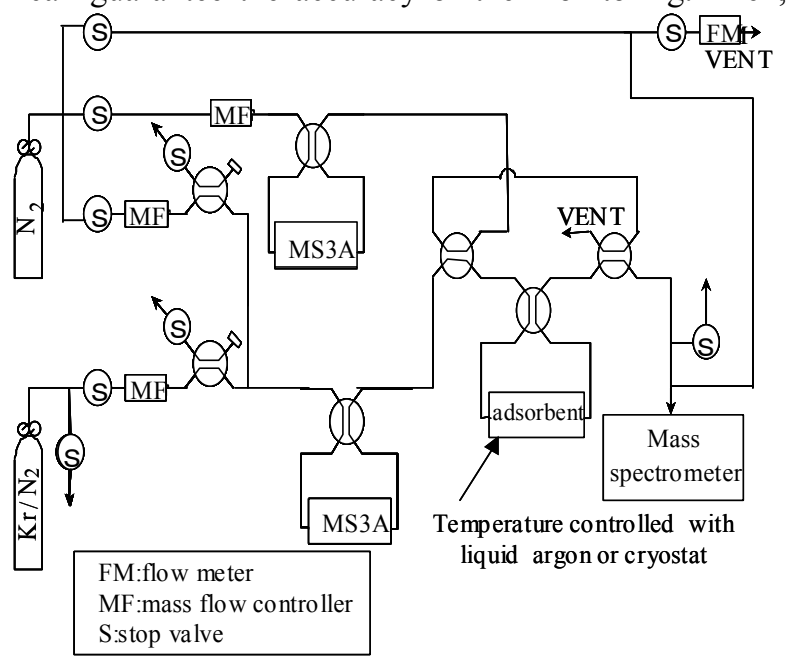

Fig. 1 Flow diagram of experimental apparatus more detailed adsorption characteristies were investigated for the adsorbents selected.

\section{EXPERIMENTAL}

The experiments were conducted by the breakthrough method. Figure 1 shows a flow diagram of the experimental apparatus used in this study. In the screening test, a tubular container made of quartz glass, which was charged with adsorbents, was placed in a constant temperature bath filled with liquid argon $(87 \mathrm{~K})$. Experiments were also conducted using liquid nitrogen $(77 \mathrm{~K})$ as a refrigerant, but condensation of nitrogen carrier gas took place. For this reason, liquid argon was used as the cooling medium. In the case of more detailed study on adsorption characteristics, a cryostat system was used to control the temperature of adsorbents at various cryogenic temperatures. In this case, adsorbents were charged in a container made of aluminum, which was attached to the cryopanel in the cryostat system. In the experiments, nitrogen containing a certain amount of krypton was introduced to the container of the adsorbents. The actual air introduced to the monitoring system of ${ }^{85} \mathrm{Kr}$ contains oxygen, carbon dioxide, water vapor and so forth, but these gases are trapped in a pre-condensation system. Thus, the major gaseous component introduced to the adsorption bed of the monitoring system is nitrogen. For this reason, nitrogen gas was used as the carrier gas. The gas flow rate was controlled with conventional mass flow controllers. The processing gases were pretreated with pre-adsorbers (MS3A) at ambient temperature to remove small amounts of water vapor that remained in the gases supplied from commercial gas bombs. The gas composition of the inlet and outlet streams of the adsorption bed was analyzed with a quadrupole mass spectrometer (Mini-Lab by MKS Inc.). The adsorbents used were preheated under nitrogen atmosphere prior to use.

\section{RESULTS AND DISCUSSION}

\subsection{Screening test of adsorbent}

Figure 2 shows the amounts of $\mathrm{Kr}$ adsorbed on various adsorbents at liquid argon temperature as the function of partial pressure of Kr. The adsorbents used were activated charcoal (produced by Kishida Reagents Chemicals Ltd.), activated charcoal

Table 1 Physical property of adsorbents

\begin{tabular}{lcccccccc}
\hline & $\begin{array}{c}\text { A.C. } \\
\text { (Kishida) }\end{array}$ & $\begin{array}{c}\text { A.C. } \\
\text { (Merck) }\end{array}$ & $\begin{array}{l}\text { Ambers- } \\
\text { orb 572 }\end{array}$ & $\begin{array}{l}\text { Ambers- } \\
\text { orb 563 }\end{array}$ & MS-5A & $\begin{array}{c}\text { Artifical } \\
\text { mordnite }\end{array}$ & $\begin{array}{c}\text { H- } \\
\text { modenite }\end{array}$ & $\begin{array}{c}\text { Ag- } \\
\text { modenite }\end{array}$ \\
\hline Particle size $(\mathrm{mm})$ & 0.39 & 0.19 & 0.45 & 0.35 & 0.86 & 0.4 & 0.4 & 0.4 \\
Packed density $\left(\mathrm{g} / \mathrm{cm}^{3}\right)$ & 0.42 & 0.37 & 0.49 & 0.53 & 0.37 & 0.265 & 0.46 & 0.58 \\
Density $\left(\mathrm{g} / \mathrm{cm}^{3}\right)$ & 0.89 & 0.835 & 0.557 & 0.712 & 0.98 & 0.98 & 0.98 & 1.31 \\
BET surface area $\left(\mathrm{m}^{2} / \mathrm{g}\right)$ & 663 & 900 & 1,100 & 550 & 415 & - & 295 & 109 \\
\hline
\end{tabular}


(produced by Merck Ltd.), Ambersorb 572 and Ambersorb 563 (produced by Sigma-Aldrich Co.), artificial mordenite (produced by Tosoh Co.), H-mordenite (prepared from natural mordenite by Kanto-Mineral Ltd.), Ag-modenite (prepared from natural mordenite by Kanto-Mineral Ltd.), and Molecular Sieve 5A (produced by Sigma-Aldrich Co.) Activated charcoals, Ambersorb 572 and Ambersorb 563 are all carbon-based adsorbents; the isotherms of these carbon based adsorbents are presented as closed symbols in Fig. 2. The Merck-produced activated charcoal adsorbent is actually used in the monitoring system of atmospheric ${ }^{85} \mathrm{Kr}$ operated by the Meteorological Research Institute in Japan. Available data on the physical property of the adsorbents used are summarized in Table 1, which indicates that the carbon based adsorbents have larger BET surface areas. As shown in Fig. 2, carbon based adsorbents have larger adsorption capacities for $\mathrm{Kr}$, and the adsorption isotherms of these adsorbents obey the Henry law when the partial pressures of $\mathrm{Kr}$ are less than $100 \mathrm{~Pa}$. The adsorption amounts of $\mathrm{Kr}$ on non-carbon based adsorbents (presented as open symbols) are considerably smaller (approximately 1 to 2 order of magnitude) than those on the carbon-based adsorbents. With regard to mordenite-based adsorbents such as the H-mordenite and Ag-mordenite adsorbents, it has been reported that the adsorption amounts of $\mathrm{Kr}$ on these adsorbents are large if the carrier gas is helium (Ianovski et al., 2002 and Munalata et al., 2000). However, the results shown in Fig. 2 indicate that the use of such adsorbents is not effective for the adsorption of $\mathrm{Kr}$ at liquid argon temperature under nitrogen atmosphere. Among the carbon based adsorbents tested, the Ambersorb 572 adsorbent has the largest adsorption capacity for $\mathrm{Kr}$ at liquid argon temperature. The major differences in the properties of synthetic carbonaceous adsorbents such as Ambersorb 572 and Ambersorb 563 are the hydrophobicity and surface area. According to data given by Sigma-Aldrich Inc. it is indicated that the Ambersorb 572 adsorbent is most hydrophilic among the Ambersorb series of adsorbents and has a larger surface area $\left(1100 \mathrm{~m}^{2} / \mathrm{g}\right)$. On the contrary Ambersorb 563 adsorbent is rather hydrophobic and has a moderate surface area $\left(550 \mathrm{~m}^{2} / \mathrm{g}\right)$. The major reason for the larger adsorption capacity of $\mathrm{Kr}$ on the Ambersorb 572 adsorbent appears to be its high surface area. However, for these carbon based adsorbents, difference in the hydrophobicity does not seem to be a major factor for the adsorption of krypton under nitrogen atmosphere.

Krypton is a non-polar molecule since it is a monatomic molecule. On the other hand, nitrogen is a polar molecule, which has probably more affinity to polar surfaces. The non-carbon based adsorbents used in this work are porous ceramic type of adsorbent (of



Fig. 2 Adsorption isotherm of $\mathrm{Kr}$ on various adsorbent at liquid argon temperature

which crystal is based on ionic bonds), and thus their surface polarity is by far greater than those of the carbon based adsorbents, which could lead to more affinity to nitrogen and less affinity to krypton. Hence, inhibition effect on the adsorption of $\mathrm{Kr}$ by coexistent nitrogen is thought to take place more greatly on the surface of the non-carbon based adsorbents.

\subsection{Adsorption isotherms of $\mathrm{Kr}$ on carbon-based} adsorbents

The result of the screening test shown above suggests that carbon-based adsorbents have larger adsorption than non-carbon-based adsorbents. Therefore, adsorption characteristics of the carbon-based adsorbents such as activated charcoal (Kishida Reagents Chemicals), activated charcoal (Merck) and Ambersorb 572 (Sigma-Aldrich) were further studied by changing adsorption temperature. In addition to these adsorbents, the Ryujyo Shirasagi G2X4/6-3 adsorbent produced by Takeda Chemical Industry Ltd. was also tested; the G2X4/6-3 adsorbent was not examined in the screening test but it was added in the study since it is used for the holdup of noble gases in nuclear power plants.

Figures 3, 4, 5 and 6 show the experimental adsorption isotherms at various temperatures on the adsorbents mentioned above. Adsorption amounts obtained with liquid argon as a coolant are slightly smaller than those at $90 \mathrm{~K}$ which was controlled with the cryostat system. This could be caused by problems 
related to temperature control with liquid argon. At the temperature of $90 \mathrm{~K}$, the adsorption amount of $\mathrm{Kr}$ on the Ambersorb 572 adsorbent was largest among the adsorbents tested, which was about 1.3 times as large as that of activated charcoal adsorbents (Merck and Kishida) and was about 2 times as large as that of the G2X4/6-3 adsorbent. However, the adsorption amount of $\mathrm{Kr}$ on the Ambersorb 572 adsorbent more remarkably decreased with increasing temperature compared with other adsorbents. The effect of temperature change on the adsorption amount of $\mathrm{Kr}$ appears to be smallest for the activated charcoal adsorbent (Kishida). For the Ambersorb 572 adsorbent, temperature control could be important when it is used in the cryogenic adsorption process, since the effect of temperature change on the adsorption amount is larger for this adsorbent.

Figures 3-6 indicate that Henry's law can be applied to the correlation of adsorption isotherms in the partial pressure range less than $100 \mathrm{~Pa}$. With regard to the Ambersorb 572 adsorbent and the activated charcoal adsorbent (Merck), experiments were conducted at the partial pressure as high as 400 $\mathrm{Pa}$ as well. As seen in Figs. 3 and 4, asymptotic behavior for adsorption amounts of $\mathrm{Kr}$ takes place in the higher partial pressure range. Therefore, the Langmuir equation was applied to the correlation of the adsorption isotherms for the Ambersorb 572 adsorbent and activated charcoal (Merck). In principle, the Langmuir equation is used to correlate the isotherms of single component adsorption. Thus, it should be noted that the effect of coexistent nitrogen is included in this correlation. The Langmuir equation is expressed as follows:

$q=K q_{s} p /(1-K p)$

$K=K_{0} \exp \left(-H_{s t} / R T\right)$

where $q, p, q_{s}, K_{0}, H_{s t}, R$ and $T$ are concentration in the solid phase $[\mathrm{mol} / \mathrm{g}]$, partial pressure of $\mathrm{Kr}[\mathrm{Pa}$, saturation adsorption amount $[\mathrm{mol} / \mathrm{g}]$, temperature independent constant $[\mathrm{mol} / \mathrm{g} \cdot \mathrm{Pa}]$, isosteric heat of adsorption in infinitesimal dilution $[\mathrm{J} / \mathrm{mol}]$, gas constant $[\mathrm{J} / \mathrm{mol} \cdot \mathrm{K}]$ and absolute temperature $[\mathrm{K}]$, respectively. When the partial pressure is small, equation (1) conforms to Henry's law that is expressed by

$q=H p$

$H=H_{0} \exp \left(-H_{s t} / R T\right)$

where $H_{0}$ is the temperature independent constant for Henry's law $[\mathrm{mol} / \mathrm{g} \cdot \mathrm{Pa}]$. For the activated characoal adsorbents (Kishida) and the G2X4/6-3 adsorbent, Henry's law was used to correlate their adsorption isotherms, since adsorption isotherm data were not taken in the higher partial pressure range for these adsorbents. The values of $q_{s}, K_{0}$, and $H_{s t}$ (Langmuir

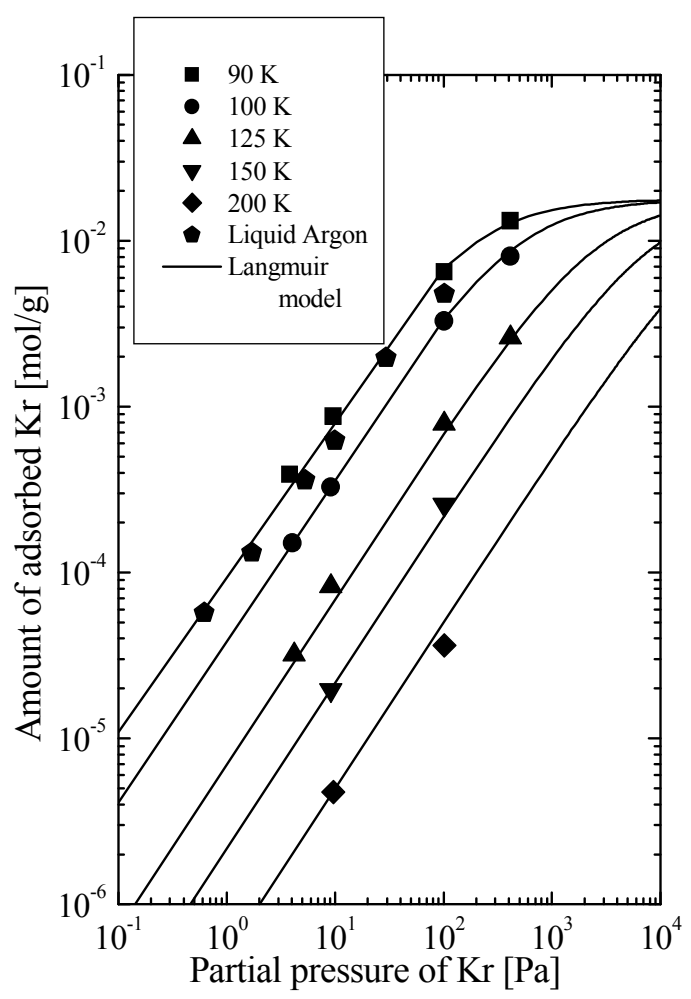

Fig. 3 Adsorption isotherm of $\mathrm{Kr}$ on Ambersorb 572



Fig. 4 Adsorption isotherm of $\mathrm{Kr}$ on activated charcoal (Merck) 
equation) or of $H_{0}$ and $H_{s t}$ (Henry's law) were optimized to correlate the adsorption isotherms by the least squares analysis. The following equations were obtained as the results of correlation.

[for Ambersor572]

$q=\frac{6.05 \times 10^{-9} p \exp (7300 / R T)}{1-3.40 \times 10^{-7} p \exp (7300 / R T)}$

[for activated charcoal by Merck]

$q=\frac{1.97 \times 10^{-8} p \exp (5990 / R T)}{1-1.23 \times 10^{-6} p \exp (5990 / R T)}$

[for activated charcoal by Kishida]

$q=2.54 \times 10^{-8} p \exp (5850 / R T)$

[for Sharasagi G2x4/6-3]

$q=1.17 \times 10^{-8} p \exp (6310 / R T)$

Solids lines in Figs. 3-6 show the results of calculation with the above equations, which indicate that the experimental adsorption isotherms are properly reproduced. As expected, the isosteric heat of adsorption is highest for the Ambersorb 572 adsorbent. The partial pressure of $\mathrm{Kr}$, which is the mixture of isotopes such as ${ }^{84} \mathrm{Kr}(56.90 \%),{ }^{86} \mathrm{Kr}(17.37 \%),{ }^{82} \mathrm{Kr}$ $(11.56 \%),{ }^{83} \mathrm{Kr}(11.55 \%)$ and so forth, in the atmosphere is about $0.1 \mathrm{~Pa}$, and small amounts of ${ }^{85} \mathrm{Kr}$ are additionally contained in the mixture. As mentioned above, the adsorption isotherms of the adsorbents obey the Henry's law at lower partial pressures, and thus the adsorption amounts of $\mathrm{Kr}$ at about $0.1 \mathrm{~Pa}$ can be estimated from the equations (5) to (8). Table 2 summaries the adsorption amount of $\mathrm{Kr}$ at the partial pressure of $0.1 \mathrm{~Pa}$ and at the temperatures of 80,90 and $100 \mathrm{~K}$ for the carbon-based adsorbents tested in this study.

Table 2 Adsorption amount of $\mathrm{Kr}$ at $0.1 \mathrm{~Pa}$

\begin{tabular}{lccc}
\hline & $\begin{array}{c}80 \mathrm{~K} \\
(\mathrm{~mol} / \mathrm{g})\end{array}$ & $\begin{array}{c}90 \mathrm{~K} \\
(\mathrm{~mol} / \mathrm{g})\end{array}$ & $\begin{array}{c}100 \mathrm{~K} \\
(\mathrm{~mol} / \mathrm{g})\end{array}$ \\
\hline Amber sorb572 & $3.5 \times 10^{-4}$ & $1.0 \times 10^{-4}$ & $3.9 \times 10^{-5}$ \\
A.C. (Merck) & $1.4 \times 10^{-4}$ & $5.2 \times 10^{-5}$ & $2.4 \times 10^{-5}$ \\
A.C. (Kishida) & $1.7 \times 10^{-4}$ & $6.3 \times 10^{-5}$ & $2.9 \times 10^{-5}$ \\
G2X4/6-3 & $1.5 \times 10^{-4}$ & $5.4 \times 10^{-5}$ & $2.3 \times 10^{-5}$ \\
\hline
\end{tabular}

\section{CONCLUSION}

A screening test of adsorbents was carried out in order to find out suitable adsorbents for the recovery of ${ }^{85} \mathrm{Kr}$ from nitrogen atmosphere. The adsorption performances of various adsorbents for $\mathrm{Kr}$ were investigated at liquid argon temperature. It was found

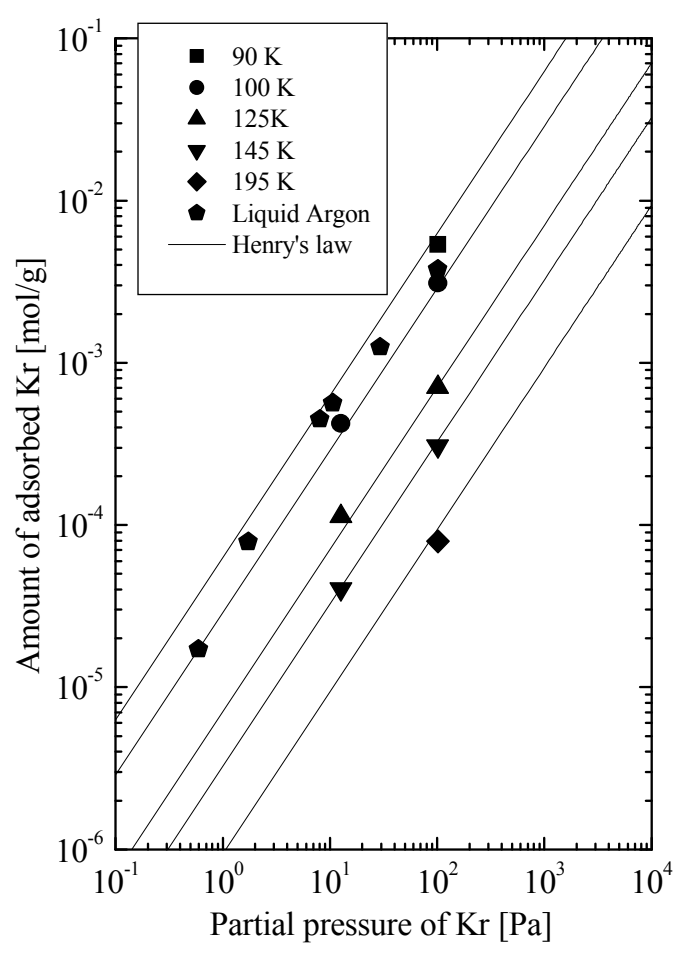

Fig. 5 Adsorption isotherm of $\mathrm{Kr}$ on activated charcoal (Kishida)

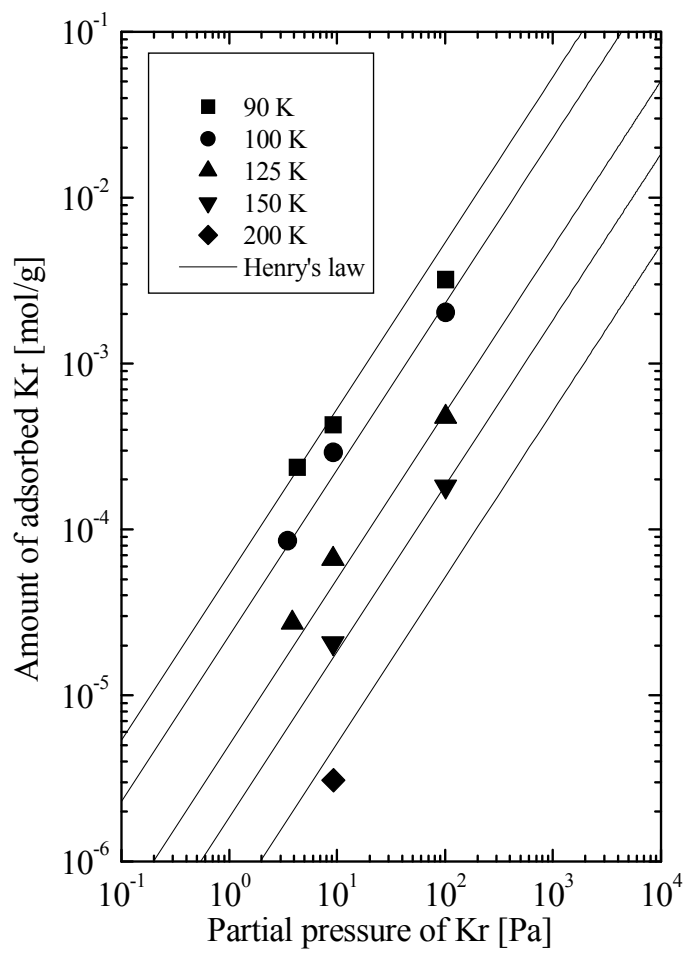

Fig 6 Adsorption isotherm of $\mathrm{Kr}$ on $\mathrm{G} 2 \mathrm{X} 4 / 6-3$ 
that carbon based adsorbents have better performance for adsorption of $\mathrm{Kr}$ and that the Ambersorb 572 adsorbent has the largest adsorption capacity. It was also found that the adsorption capacity of non-carbon based adsorbents is much lower than that of carbon-based adsorbents.

More detailed adsorption characteristics were investigated for carbon-based adsorbents using the cryostat system. In the temperature range of 90-200 K, the Ambersorb 572 adsorbent has the largest adsorption capacity for the adsorption of $\mathrm{Kr}$ under nitrogen atmosphere. The effect of temperature change on the adsorption capacity was found to be largest for the Ambersorb 572 adsorbent and smallest for the Kishida-produced activated charcoal adsorbent.

\section{REFERENCES}

Hirota, M., et al., J. Radiat. Res., 45, 405 (2004).

Ianovski, D., et al.: J. of Nucl.Sci. and Technol., 39[11], 1213 (2002).

Munakata K., et al.: J. of Nucl.Sci. and Technol., 36[ 9], 818 (1999).

Munakata K., et al.: J. of Nucl.Sci. and Technol., 37[1],84 (2000).

Munakata, K., et al., J. Chem. Eng. Jpn., 34[7], 853

(2001). 